\title{
Frühe menschliche Spuren in der subalpinen Stufe des Hinterrheins
}

Ur - und frühgeschichtliche Funde in Form von Siedlungsplätzen, Grabfeldern, Einzelgräbern mit und ohne Beigaben, Streufunden und Schalensteinen in Alt Fry Rätien geben unter anderem Auskunft über die Erstbesiedlung der Alpentäler durch den Menschen. W. BURKART hat zur Erhellung der Ur- und Frühgeschichte Rätiens durch Deutung und Sicherstellung zum Vorschein gekommener Funde sowie durch Grabungsarbeiten ganz wesentliche Beiträge von internationalem Rang geleistet. Im Zuge des Nationalstrassenbaus wurden besonders in der Mesolcina weitere ur - und frühgeschichtliche Funde getätigt (ERB \& SCHWARZ 1969). *) Aus der Jungsteinzeit sind im Schams und Rheinwald bisher keine Funde gemacht worden. Auf dem Petrushügel bei Cazis wurde 1930 von BURKART die einzige neoli thische Siedlung im Kt. Graubünden entdeckt. *) Es handelt sich hier um eine befestigte Höhensiedlung von etwa 2000 v. Chr., deren Bewohner Jagd, Viehzucht und Feldbau sowie Handel mit Steinbeilen und -Meisseln längs des Rheins betrieben (ERB \& SCHWARZ 1969, TSCHUMI O., 1947). Gräberfelder und Einzelbestattungsplätze in Clugin, Donatz, Lohn, Mathon, Wergenstein, Patzen, Zillis, Andeer und Bärenburg sowie das frühbronzezeitliche Kuppelgrab von Donath, dem ältesten bronzezeitlichen Fund Graubündens, lassen auf eine frühbronzezeitliche Besiedlung des Schams schliessen, während das obere Hinterrheingebiet bis zu Beginn der Römerzeit nur Durchzugsland war (ERB \& SCHWARZ, 1919, MANI B. 1958).

Als mittelbronzezeitlich werden Funde eines Beiles bei Pignia-Bad bei Andeer, einer etwas jüngeren Schmucknadel in der Nähe des Lai da Vons und zweier Waffen bei Splügen bezeichnet (ERB \& SCHWARZ, 1969). Aus der Aelteren Eisenzeit fehlen zwischen der Via Mala und San Bernadino jegliche Spuren. Jungeisenzeitliche Funde wurden im Schams und im Rheinwald gemacht (ERB \& SCHWARZ, 1969). (Vgl. Abb. 1)

Nach der Darstellung von W. BURKART (1953) drangen um $2000 \mathrm{v}$. Chr. erstmals Menschen vom Rheintal hinauf nach Rätien. Um 1700 v.Chr. fand die Einwanderung der frühbronzezeitlichen Menschen aus dem Donauraum in einige Alpentäler Bündens statt (Lugnez, Oberhalbstein, En- gadin). Im 6. vorchristlichen Jahrhundert erfolg te von Osten der Zustrom des eisenzeitlichen Rätervolkes ins Rheingebiet und Engadin sowie die Kelteninvasion vom Tessin ins Misox nordwärts bis ins Bündner Oberland. Im Rahmen einer Diplomarbeit am Geographischen Institut der Universität Zürich lieferte eine zwecks Datierung von spätglazialen Moränenwällen am Lai da Vons (1991 m) durchgeführte Pollenanalyse überraschenderweise auch Hinweise zur ersten menschlichen Besiedlung des Hinterrheingebiets, die nun im folgenden näher beschrieben und gedeutet werden sollen. Im Pollendiagramm des Lai da Vons treten die ersten menschlichen Spuren in etwa $140 \mathrm{~cm}$ Tiefe auf im Jüngeren Atlantikum (Pollenzone VII), also zu Beginn der Jungsteinzeit um $2820 \mathrm{v}$. Chr. (vgl. Abb. 2). Hier erreicht die Getreidekurve mit 4\% ein erstes Maximum (festgestellt wurden u. a. Pollen von Roggen) bei $84 \%$ Nichtbaumpollen. Erstmals treten Holzkohlestückchen auf. Der Spitzwegerich, der "Fusstritt des Weissen Mannes", ein besonders für Nordeuropa herangezogener Kulturzeiger, setzt wenig tiefer bei $155 \mathrm{~cm}$ ein. In $150 \mathrm{~cm}$ Tiefe fand sich zudem ein nicht näher bestimmbares Getreidekorn. Funde von Pollenkörnern des Leins, die erstmals in $170 \mathrm{~cm}$ auftreten, werden ebenfalls in $150 \mathrm{~cm}$ Tiefe häufiger. Der Lichtungszeiger Selaginella Selaginoides (Moosfarn) steigt an auf Werte von über $100 \%$ der Pollensumme I. Rodungszeiger, wie zungen-und röhrenblütige Körbchenblütler, Wegerich sowie alle Farnsporen-Kurven steigen synchron mit deutlichem Knick an (Aus Abb. 2 nicht ersichtlich, da nur das Hauptdiagramm des Postglazials hier abgebildet ist. Vollständiges Diagramm in:BURGA C. A. , 1975, unveröffentlichtes Manuskript Universität Zürich). Zudem tritt in ca. $140 \mathrm{~cm}$ Tiefe eine sandige Torflage auf, die auf verstärkte Erosion während einer Rodungstätigkeit hindeuten kann. Der Nichtbaumpollengipfel in $142 \mathrm{~cm}$ Tiefe wurde mit $4770+90$ Jahre vor heute radiometrisch datiert.

C. A. Burga, dipl. nat. , Assistent an der Botanischen Anstalt der Universität Basel, Schönbeinstr. 6, 4056 Basel. 
Abb. 1: Ur -und frühges chichtliche Fundstellen im Domleschg, Schams, Rheinwald und Misox sowie frühgeschichtliche Routen von Chur bis nach Mesocco (Stand 1975) (Zusammengestellt nach BURKART W. (1953), CAMINADA C. (o. J.), ERB H. und SCHWARZ G. T. (1969), MANI B. (1958) und Bündnerzeitung vom 31. Januar 1975)

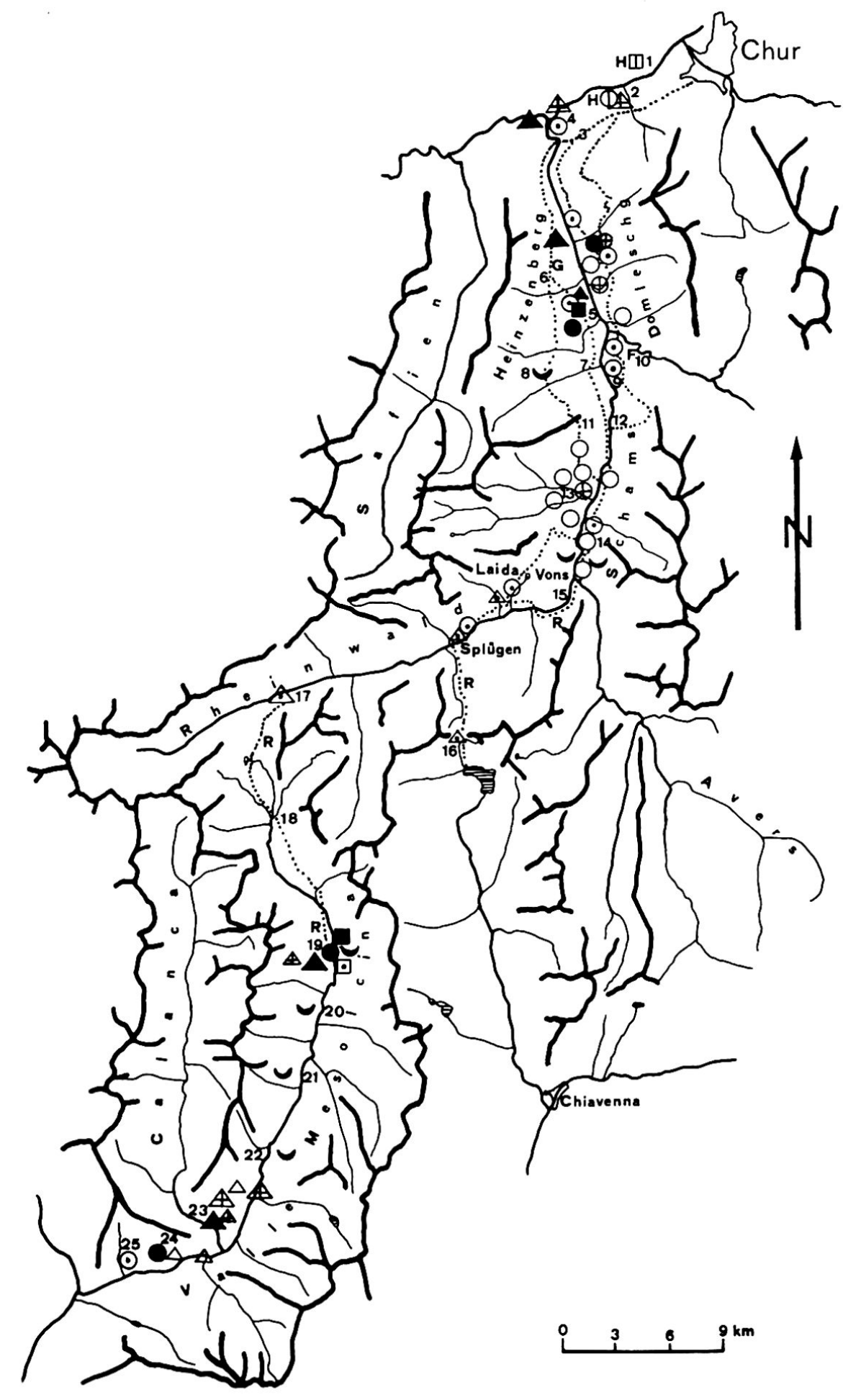

Ortschaften / Lokalitäten :

1 Felsberg

2 Domat/Ems

3 Vogelsang

4 Reichenau / Tamins

5 Cazis

6 Präz

7 Thusis

8 Urmein

9 Hohenrätien

10 Carschenna

11 Summapunt

12 Via Mala/Rongellen

13 Donath

14 Andeer

15 Roffla

16 Splügenpass

17 Hinterrhein

18 San Bernardino

19 Mesocco

20 Soazza

21 Cabbiolo

22 Sorte

23 Castaneda

24 San Vittore

25 Monticello

\section{$\square \quad$ Jungsteinzeit \\ Bronzezeit \\ $\triangle \quad$ Eisenzeit \\ $\triangle$ Siedlung \\ $\bullet \odot \Delta$ Streufund(e)/Einzelfund \\ 田丹 Gräberfeld}

(1) $\Delta$ Einzelgräber

$\square \bigcirc \triangle$ Einzelgräber oder Grabfeld ohne Beigaben, Alter unsicher

F Felszeichnung

G Grabstein mit lepontischer Inschrift

H Höhle

Zeichen- und Schalenstein

Ur- und frühgeschichtliche Routen, z. T. römerzeitlich begangen

...... Römische Strassen und Wege, z. T. noch fraglich 
Abb. 2: LAI DA VONS (GR) $1991 \mathrm{~m}$ koord. 749.130/161240

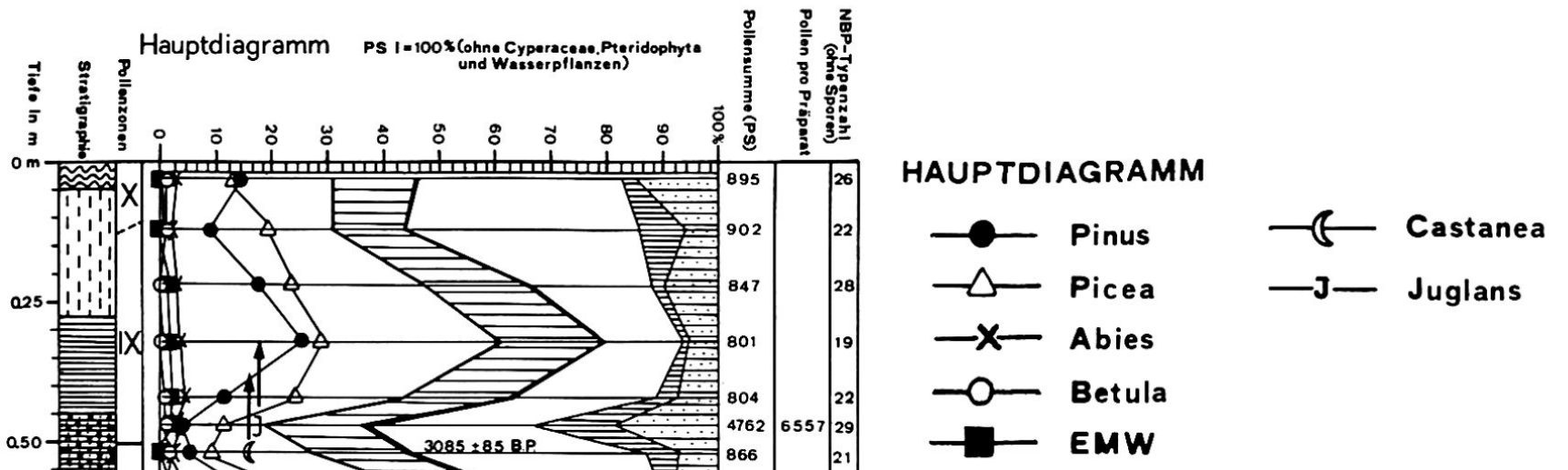

STRATIGRAPHIE

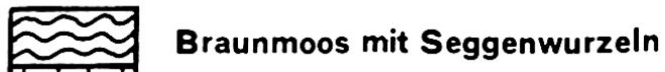

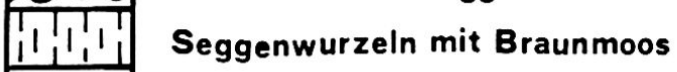
Seggenwurzeln mit Wollgras Seggenwurzeln mit Braunmoos und Wollgras

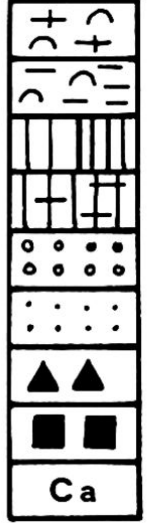

Kalkmudde mit Erbsenmuscheln

Seekreide

Blaugrauer bzw. gelbgrauer Ton

Blaugrauer Ton hell marmoriert

Sand $2000 \mu-60 \mu$

Silt, Ton $<60 \mu$

$\leq 5 \mathrm{~mm}$

$\leq 12 \mathrm{~mm}$

Karbonat

weisse und schwarze Kalke * weisse, graue und gelbe Dolomite Grünschiefer

Kluftquarz 
Alle diese Indizien drängen zur Frage, ob nicht schon früher als bisher angenommen im Schams und vorderen Rheinwald möglicherweise jungsteinzeitliche Menschen, wenn auch nur saisonal, hausten. Die Holzkohlenfunde allein würden kaum einen solchen Schluss erlauben, da bekanntlich ausser dem Menschen auch der Blitzschlag Feuer entfachen kann. Jedoch treffen hier mehrere verschiedenartige Hinweise zusammen, die wohl eine Annahme früherer Besiedlung, wenn auch nur zur warmen Jahreszeit, als berechtigt erscheinen lässt.

Vorliegende Holzkohlenfunde könnten auch in Zusammenhang mit Opferhandlungen an den Quellen des Lai da Vons gebracht werden, zumal der See an der alten Umgehungsroute westlich der Rofflaschlucht liegt. C. CAMINADA (o. J.) weist auf diese Form des Wasserkultus hin, der sicher bis in die Bronzezeit zurückreichen soll, wie bronzene Votivgaben von St. Moritz, Ruis und Parpan (BERTOGG H. , 1943)dies bezeugen. Es ist also wohl mehr als Zufall, dass bei Quellenfassungen und bei Wasserläu fen Bronzebeile gefunden wurden, so bei IlanzSt. Martin, in Pignia-Bad bei Andeer, oberhalb Parpan und in St. Moritz (CAMINADA o. J.,ERB H. briefl. Mitt. 1975).

"Aber noch kann die umfassende, kritische Urund Frühgeschichte dieses alpinen Transitlandes nicht geschrieben werden"bemerkt ERB H. (1969), und viele alte Siedlungsplätze harren noch ihrer Entdeckung (BURKART W. , 1953). Dazu gehört auch die Deutung des geheimnis vollen Kreises der Zeichen - und Schalensteine aus dem Bergell, Prättigau, Hinterrheintal, Vorderrheingebiet und Misox, die mit Blutopfern und alten Sagen (BERTOGG H. , 1943), Harz-und Butterlichter sowie Astronomie (Kalendersteine)(CAMINADA C. o. J.) in Zusammenhang gebracht werden.

Urgeschichtliche Hinweise geben auch rätoromanische Sagen und Märchen, Sprichwörter und Anordnungen aus der Volksthedizin, die in bewundernswerter Weise von DECURTINS C.

(1896 bis 1919, 13 Bde.) in seiner Rätoromanischen Chrestomathie gesammelt wurden, sowie die Deutung von Flurnamen und Ortsbezeichnungen.

Abschliessend sei festgehalten, dass aufgrund der oben dargelegten Fakten mit einem frühe- ren als bisher geglaubten Auftreten des Menschen im Schams und vorderen Rheinwald gerechnet werden darf. Freilich muss dieser Befund durch weitere Funde im Hinterrheingebiet gestützt und bestätigt werden.

\section{LITERATUR:}

Bertogg H. 1943 Aus der Welt des rätischen Heidentums in: Bündner $\mathrm{Ka}$ lender 1943, S. 8-16

Burga C. A. 1975 Spätglaziale Gletscherstände im Schams. Eine glazialmorphologisch-pollenanalythische Untersuchung am Lai da Vons (GR). Unveröff. Manuskript Univ. Zürich.

Burkart W. 1942 Das Kuppelgrab bei Donath Bündner Monatsblatt Dez. 1942.

1953 Die urgeschichtliche Besiedlung Alträtiens. Bündner Schulbl. Jg.13,Nr.2,S.67-107

Bündner 1975 Nationalstrasse auf steinZeitung zeitlichem Niveau (31.1.75)

CaminadaC.o.J. Die verzauberten Täler.Die urgeschichtlichen Kulte und Bräuche im alten Rätien.

Decurtins C.1896 Rätoromanische Chrestomabis 1919 thie 13 Bde.

Erb H. $\quad 1975$ Briefliche Mitteilung

Erb H. \& Die San Bernardinoroute

Schwarz G.T. von der Luzisteig bis in die Mesolcina in ur- und frühgeschichtlicher Zeit. Schriftenreihe des Rhätischen Museums Chur, Heft 5.

Mani B. 1958 Heimatbuch Schams.

Tschumi O. 1947 Urgeschichte der Schweiz Bd. 1

ANMERKUNG *): Die bis jetzt älteste prähist. Siedlung in GR wurde 1975 während des Nationalstrassenbaus im Castello Mesocco (Mesocco) entdeckt. Sie wird mit etwa 5000 Jahren var Chr. datiert, also Uebergang von der mittleren zur jüngeren Steinzeit. (BZ, Jan. 1975) 